\title{
Microcantilever equipped with nanowire template electrodes for multiprobe measurement on fragile nanostructures
}

\author{
Lin, Rong; Bøggild, Peter; Hansen, Ole
}

Published in:

Journal of Applied Physics

Link to article, DOI:

$10.1063 / 1.1756214$

Publication date:

2004

Document Version

Publisher's PDF, also known as Version of record

Link back to DTU Orbit

Citation (APA):

Lin, R., Bøggild, P., \& Hansen, O. (2004). Microcantilever equipped with nanowire template electrodes for multiprobe measurement on fragile nanostructures. Journal of Applied Physics, 96(5), 2895-2900.

https://doi.org/10.1063/1.1756214

\section{General rights}

Copyright and moral rights for the publications made accessible in the public portal are retained by the authors and/or other copyright owners and it is a condition of accessing publications that users recognise and abide by the legal requirements associated with these rights.

- Users may download and print one copy of any publication from the public portal for the purpose of private study or research.

- You may not further distribute the material or use it for any profit-making activity or commercial gain

- You may freely distribute the URL identifying the publication in the public portal

If you believe that this document breaches copyright please contact us providing details, and we will remove access to the work immediately and investigate your claim. 


\title{
Microcantilever equipped with nanowire template electrodes for multiprobe measurement on fragile nanostructures
}

\author{
R. Lin, P. Bøggild, and O. Hansen \\ Department of Micro- and Nanotechnology, Technical University of Denmark, Building 345 East, \\ DK-2800 Lyngby, Denmark
}

(Received 9 February 2004; accepted 6 April 2004)

\begin{abstract}
We present a technique for fabricating nanoelectrode extensions to microcantilevers for multiprobe electrical characterization. For electrical measurements of fragile samples, such as thin films and nanostructures, it is advantageous to combine a small contact force with a small contact area, which can be done by reducing the dimensions of the electrodes to nanoscale dimensions. Here we report a fabrication method of a nanoscale four-point probe utilizing silicon nanowires as templates for metal electrodes. Using nanomanipulation, we attach 200-300 $\mathrm{nm}$ wide silicon nanowires to microfabricated cantilevers. By subsequently covering these nanowires with a metallic coating, they are made conducting and at the same time fixed to the cantilevers. These silicon nanowire four-point probes were tested on 7 and $35 \mathrm{~nm}$ thick Au films as well as poorly adhering $16 \mathrm{~nm}$ thin Au nanowires deposited on a silicon surface through a nanofabricated shadow mask. It was found that the nanowire extensions dramatically reduce the damage of the studied samples, while nearly reproducing the resistivity measurements of the unmodified, but more destructive micro four-point probes. (C) 2004 American Institute of Physics. [DOI: 10.1063/1.1756214]
\end{abstract}

\section{INTRODUCTION}

With continuing advances in microelectronic device miniaturization, there is a need for experimental methods for investigating metallic or semiconducting nanowires and thin films fabricated in various ways, as potential components and interconnects in device applications. To study the electrical properties of such nanowires, contacting methods including lithographically fabricated in-plane electrodes fixed on the surface ${ }^{1-3}$ or conducting atomic force microscopy $y^{4,5}$ have been employed. Lithographically defined electrodes connecting the nanowires to macroscopic electrodes allow both two- and four-point resistance measurements to be performed. Such in-plane electrodes are however fixed with respect to the wires, and are thus not as convenient for electrical characterization as a positionable probe. With conducting atomic force microscopy it is possible to measure the resistance of nanowires and thin films directly at different positions with a high spatial resolution. Such measurements are however typically performed in a two-point configuration, with one part of the nanostructures connected to a fixed electrode, and the SPM acting as the second electrode in a twopoint configuration. For small electrode dimensions the contact resistance inherently influencing the two-point measurement may produce errors that are difficult to estimate and thus compensate for. Four independent scanning probes is a method for eliminating the contact resistance while keeping the positionability, ${ }^{6}$ which is however technically complicated and inconvenient to use.

The development of silicon-based microscale four-point probe ( $\mu 4 \mathrm{PP})$ technique has opened a convenient possibility to study the electronic properties of samples on a small scale, ${ }^{7}$ thin films, ${ }^{8}$ and surface layers of semiconducting surface. ${ }^{9,10}$ These probes can quickly be moved anywhere on the sample, enabling small regions and isolated domains to be measured. ${ }^{11}$ Direct resistance measurements using $\mu 4$ PPs with different electrode spacing on Au and Pt nanowires fabricated using a nanofabricated shadow mask ${ }^{12}$ have been performed. ${ }^{13}$ However, one severe problem encountered during $\mu 4 \mathrm{PP}$ resistance measurements on fragile nanowires was sample damage inflicted by the probe, as shown in Fig. 1. Upon contact, thin $\mathrm{Au}$ nanowires may be completely or partly removed by the probe. The contact forces are in the $\mu \mathrm{N}$ range, depending on the dimensions of the microcantilevers. Another issue is that the microcantilevers block the view of the contact points and the nanowire, making precise positioning on the nanowire difficult.

To overcome this problem, we considered several methods for reducing the diameter of the electrodes to a size that is comparable to or smaller than the dimensions of the nanowire. With narrower electrodes, the diminishing contact area inevitably leads to larger contact pressures. However, since the spring constant of the electrode scales with the electrode diameter to the fourth power, while the contact area can be taken to scale with the electrode diameter squared, more slender electrodes generally should lead to less severe sample damage in more confined areas. Besides, sharper, narrower tips allow better line of sight of contact point.

Electron-beam deposition technique was previously used to grow narrow tips at the apexes of a micro four-point probe. ${ }^{11}$ Although very narrow gaps down to $100 \mathrm{~nm}$ can be made with this method, electrodes more than a few microns long are excessively time consuming to make. Here we demonstrate an alternative method for fabrication of nanoprobes using long prefabricated single-crystalline silicon nanowires with diameters of 200-300 $\mathrm{nm}$. We present two- and fourpoint resistance measurements on Au thin films with differ- 


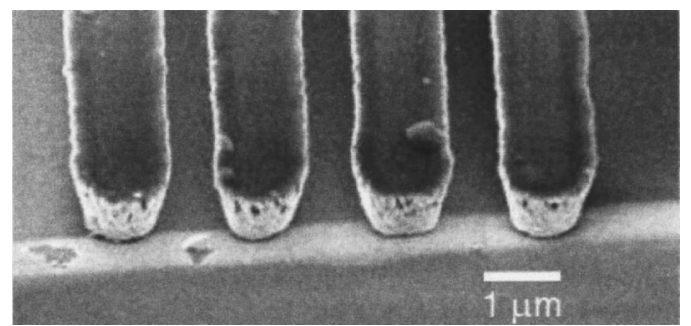

FIG. 1. SEM image showing a micro four-point probe in contact with an Au wire, after a previous measurement $1 \mu \mathrm{m}$ to the left. The Au wire is partly stripped off in $\sim 500 \mathrm{~nm}$ size areas, in two of the four measurement points.

ent thickness and two-point measurement on $\mathrm{Au}$ nanowires using these probes.

\section{EXPERIMENT}

The experimental setup used for both manipulation and conductance measurements is a home-built microprobe measurement station. The setup consists of a Newport XYZ translation stage with $100 \mathrm{~nm}$ accuracy, a Burleigh PCS5400 micromanipulator with a resolution of $50 \mathrm{~nm}$, and a high-resolution optical Navitar microscope lens equipped with a camera, all mounted on an air-damped optical table. The probe stage mounted on the Burleigh micromanipulator tilts the probe at an angle of $10-30^{\circ}$ with respect to the surface. A small combined current source and instrumentation amplifier is mounted a few centimeters from the probe to reduce electromagnetic noise picked up in cables. During measurement, an ac current with frequency in the range of $100-300 \mathrm{~Hz}$ was applied to the outer electrodes, while the signal from the inner probes was amplified close to the probe by the integrated source meter, and picked up by a Stanford SR 830 lock-in amplifier. The $I / V$ curve was continuously monitored using an oscilloscope to verify that ohmic contact was established at all times. For the two-point measurement, a computer-controlled Keithley 2400 source meter was used. To prevent excessive joule heating in the nanoprobes and nanowire the current was in all cases kept below $5 \mu \mathrm{A} .{ }^{14}$

The single-crystalline silicon nanowires (SiNWs), provided by Dr. Erik Bakkers at Philips Research Laboratories, ${ }^{15}$ typically have diameters of 200-300 nm and lengths of 40-100 $\mu \mathrm{m}$. Single-crystalline Si nanowires have a high elastic modulus of $129.5 \mathrm{GPa}$ in the [100] direction and $186.5 \mathrm{GPa}$ in the [111] direction, ${ }^{16}$ compared to silicon dioxide, $74 \mathrm{GPa},{ }^{16}$ and to gold, $80.6 \mathrm{GPa} .{ }^{17}$ The micro fourpoint probes ( $\mu 4 \mathrm{PPs})$, fabricated using conventional siliconbased microfabrication technique, consist of four metallized $\mathrm{SiO}_{2}$ microcantilevers extending over the edge of a silicon support chip. Probes with different electrode pitch, ranging from 60 down to $1.1 \mu \mathrm{m}$, have previously been fabricated. ${ }^{7}$

In this work, such $\mu 4 \mathrm{PP}$ was used as a multifinger manipulation tool to pick and place SiNW on other micro fourpoint probe cantilevers. The fabrication process is schematically illustrated in Figs. 2(a)-2(c). First we use a $\mu 4 \mathrm{PP}$ to pick up a single nanowire extending from the Si substrate, and subsequently to place it onto another $\mu 4 \mathrm{PP}$, the "target" $\mu 4$ PP. The optical image in Fig. 2(d) shows the fourth wire being placed onto the empty cantilever of the target probe,
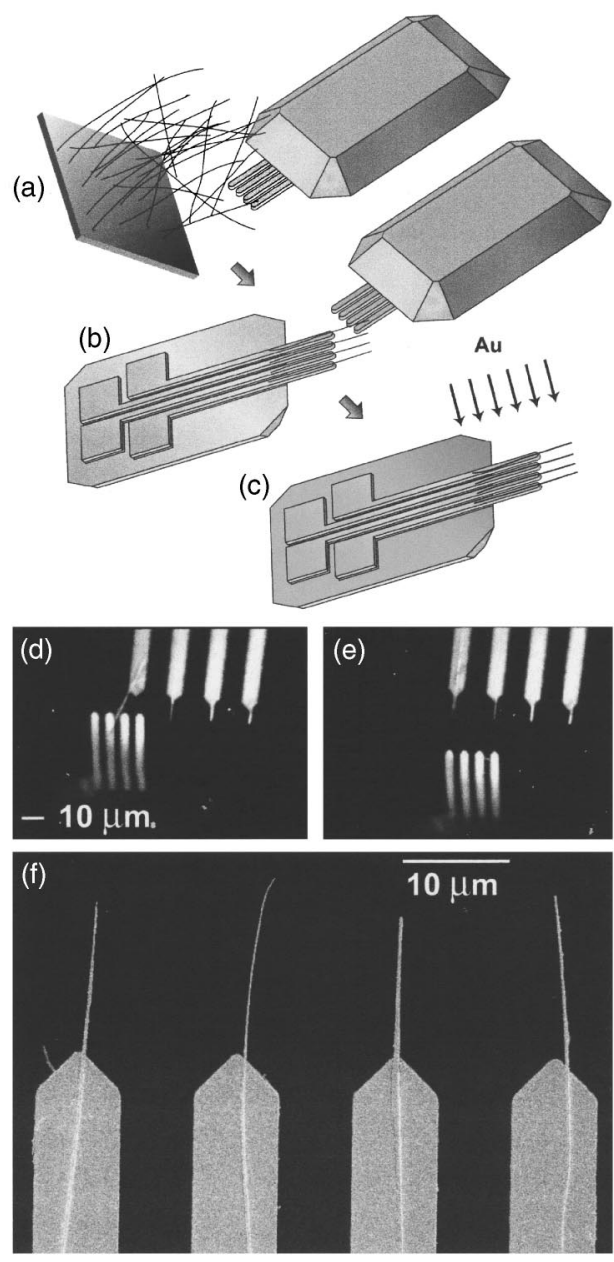

FIG. 2. (a) Illustration of one 4PP picking up a SiNW from a silicon substrate. (b) The first 4PP is manipulating the nanowire onto the target 4PP cantilever. (c) Deposition of Ti/Au covers the nanowires with a conducting layer, while at the same time fixing the wires to the electrodes. (d) and (e) Optical images depicting a $\mu 4 \mathrm{PP}$ (bottom) being used to place a Si nanowire on one of the microcantilevers of the target 4PP (top). (f) A SEM image of a micro four-point probe equipped with nanowire nanoelectrodes. The scale bars are $10 \mu \mathrm{m}$.

on which three nanowires have already been positioned. Figure 2(e) shows an optical image of a completed SiNW fourpoint probe (SiNW-4PP) prior to metallization. In the assembly process, positioning single wires on top of cantilevers is relatively fast, compared to parallel alignment of the nanowires as well as fine tuning of the length of the suspended part of the nanowires.

To make the nanowires conducting, an Au layer with a thickness between 70 and $270 \mathrm{~nm}$ was deposited with a 30 $\mathrm{nm}$ thick Ti layer used as an adhesion layer. By embedding the wires in this metallic coating layer, sufficient adhesion to the cantilevers is ensured. Figure 2(f) displays a scanning electron microscope (SEM) image of a SiNW-4PP assembled on a microprobe with an electrode pitch of roughly $16 \mu \mathrm{m}$, and the metallic coating layer covering the Si wire on the side facing up. The width of each metallized nanoelectrode is roughly determined by the width of the associated nanowire template. In Table I we give the specifications of all the micro and SiNW four-point probes used in this work. 
TABLE I. Specifications of the micro and a nanowire four-point probes used in this work. $M$ indicates a micro four-point probe $\mu 4 \mathrm{PP}$ while $N$ indicates a silicon nanowire four-point probe, SiNW-4PP.

\begin{tabular}{lccc}
\hline \hline Probe & $\begin{array}{c}\text { Metal coating } \\
(\AA \mathrm{A} \text { Ti/Au })\end{array}$ & $\begin{array}{c}\text { Interelectrode } \\
\text { spacing }(\mu \mathrm{m})\end{array}$ & $\begin{array}{c}\text { Probe geometry } \\
\text { factor } K\end{array}$ \\
\hline$M 1$ & $100 / 1000$ & 16 & 1.00 \\
$M 2$ & $100 / 1000$ & 3 & 1.00 \\
$M 3$ & $100 / 1000$ & 2 & 1.00 \\
$N 1$ & $300 / 2700$ & $29 / 15 / 19$ & 1.39 \\
$N 2$ & $300 / 1500$ & $11 / 16 / 21$ & 0.95 \\
$N 3$ & $300 / 1500$ & $21 / 17 / 14$ & 1.00 \\
$N 4$ & $300 / 700$ & 16 & N/A $(2 \mathrm{PP})$ \\
$N 5$ & $300 / 700$ & 16 & N/A (2PP) \\
\hline \hline
\end{tabular}

All the micro four-point probes are fabricated with equispaced probe tips with the interelectrode spacing $s$. When these probes are used for measurements on infinitely thin films, the measured resistance $R=V / I$ is related to the thin film resistivity $\rho=\pi / \ln 2(V / I) t$, where $t$ is the sample film thickness and $\pi / \ln 2$ is a geometry factor.

The SiNW four-point probes are unfortunately not equispaced; probe $N 1$, for instance, have a spacing of $29 \mu \mathrm{m}$ between the first and second electrode, while just $15 \mu \mathrm{m}$ between the center electrodes, as shown in Table I. The relation between the measured four-point resistance and the thin film resistivity must in the case of unequal electrode spacings, $s_{1}, s_{2}$ and $s_{3}$, include an additional probe geometry factor ${ }^{18} K=\ln (4) / \ln \left[\left(s_{1}+s_{2}\right)\left(s_{2}+s_{3}\right) /\left(s_{1} s_{3}\right)\right]$. Moreover, samples of finite dimensions comparable to the size of the probe must be corrected for by multiplying with a sample geometry factor $c$. Values of $c$ for different sample dimensions and geometries are given in Ref. 19. In the case of an infinite sheet the sample geometry factor is $c=1$. Correcting for sample geometry and probe geometry, the relation between four-point resistance and thin film resistivity becomes

$$
\rho=c K \frac{\pi}{\ln 2} \frac{V}{I} t
$$

In Table I, we have listed the probe geometry factor $K$ for all the probes used.

The samples on which electrical measurements were performed in this work were fabricated by electron beam evaporation of thin metal films. The nanowires were deposited through a nanostencil mask, which allows for easy, resistfree fabrication of nanostructures. The nanostencils were fabricated on a $150 \mathrm{~nm}$ thick silicon nitride membrane using focused ion beam etching. We measured on four types of samples: (A) $35 \mathrm{~nm}$ thick Au pad with lateral dimensions of $60.8 \times 19.0 \mu^{2}$, (B) $35 \mathrm{~nm}$ thick Au thin film, (C) $7 \mathrm{~nm}$ thick $\mathrm{Au}$ film, and (D) $16 \mathrm{~nm}$ thick $\mathrm{Au}$ nanowire of lateral dimensions $250 \times 1.1{\mu \mathrm{m}^{2}}^{2}$. For convenience, the measurements performed on thin-film samples are numbered ( $m 1, m 2, \ldots, m 11)$, see Table II. Prior to Au deposition on the $1 \mu \mathrm{m}$ thick $\mathrm{SiO}_{2}$ substrate, a $10 \mathrm{~nm}$ thick Ti layer was deposited to enhance the adhesion to the substrate.

\section{MEASUREMENTS ON THIN FILMS}

We characterized the micro four-point probes by measuring the resistance of a $35 \mathrm{~nm}$ thick Au pad both in two-point (2PP) and 4PP configuration. Two probes with different interelectrode spacing, 3 and $16 \mu \mathrm{m}$, were used and the results are summarized in Table II. Measuring in a two-point configuration, a resistance of $126.65 \pm 5.47 \Omega$ and $112.80 \pm 0.50$ $\Omega$ was found for probe $M 1(m 1)$ and $M 2(m 2)$, respectively.

In a four-point configuration, both the probe geometry factor $K$, as well as the sample geometry factor $c$ (see Tables I and II), must be taken into account for correctly calculating the resistivity using Eq. (1). With both probes $M 1(m 4)$ and $M 2(m 5)$, the resistivity was found to be a factor of approximately three times larger than the bulk resistivity for gold, $\rho_{\text {bulk }}=2.2 \times 10^{-8} \Omega \mathrm{m}$.

The difference in the measured values between the $2 \mathrm{PP}$ and the 4PP configurations is of the order of $100 \Omega$ for both probes. This corresponds roughly to the serial resistance of the gold film on each of the two microchip electrode struc-

TABLE II. Measurement results on three thin film samples: (A) $35 \mathrm{~nm}$ thick Au pad measured with both $\mu 4 \mathrm{PP}$ and SiNW-4PP. The sample correction factor $c$ for the 4PP measurements was found from the sample dimensions $\left(60.8 \times 19.0 \mu \mathrm{m}^{2}\right)$ relative to the probe spacing of probes $M 1(16 \mu \mathrm{m})$ and $M 2(3 \mu \mathrm{m})$, using the tables in Refs. 12 and 19. (B) $35 \mathrm{~nm}$ thick Au film. (C) $7 \mathrm{~nm}$ Au film measured with both $\mu 4 \mathrm{PP}$ and SiNW-4PPs. In the table the measurements performed are numbered as $m 1, m 2, \ldots, m 11$.

\begin{tabular}{|c|c|c|c|c|c|c|}
\hline Sample & Probe & Measurements & Configuration & $\begin{array}{l}\text { Resistance } \\
R=V / I \Omega\end{array}$ & $\begin{array}{l}\text { Sample correction } \\
\text { factor } c^{\mathrm{a}}\end{array}$ & $\begin{array}{c}\text { Resistivity }(\mu \Omega \mathrm{cm}) \\
\rho=c K(\pi / \ln 2) R t\end{array}$ \\
\hline \multirow[t]{5}{*}{ A } & $M 1$ & $m 1$ & $2 \mathrm{PP}$ & $126.6 \pm 5.5$ & & \\
\hline & $M 2$ & $m 2$ & $2 \mathrm{PP}$ & $112.8 \quad \pm 0.5$ & & \\
\hline & $N 4$ & $m 3$ & $2 \mathrm{PP}$ & $141.2 \pm 0.3$ & & \\
\hline & $M 1$ & $m 4$ & $4 \mathrm{PP}$ & $1.53 \pm 0.05$ & 0.253 & 6.139 \\
\hline & $M 2$ & $m 5$ & $4 \mathrm{PP}$ & $0.383 \pm 0.040$ & 0.844 & 5.131 \\
\hline \multirow[t]{4}{*}{ B } & $M 1$ & $m 6$ & $4 \mathrm{PP}$ & $0.237 \pm 0.005$ & 1 & 3.759 \\
\hline & $N 1$ & $m 7$ & $4 \mathrm{PP}$ & $0.222 \pm 0.005$ & 1 & 4.893 \\
\hline & $N 2$ & $m 8$ & $4 \mathrm{PP}$ & $0.200 \pm 0.009$ & 1 & 3.01 \\
\hline & $N 3$ & $m 9$ & $4 \mathrm{PP}$ & $0.203 \pm 0.006$ & 1 & 3.213 \\
\hline \multirow[t]{2}{*}{$\mathrm{C}$} & $M 1$ & $m 10$ & $4 \mathrm{PP}$ & $1.854 \pm 0.012$ & 1 & 5.88 \\
\hline & $N 1$ & $m 11$ & $4 \mathrm{PP}$ & $1.956 \pm 0.009$ & 1 & 8.61 \\
\hline
\end{tabular}

${ }^{\mathrm{a}}$ Reference 20 . 
tures leading from the bonding pads to the surface. ${ }^{7}$ The spreading resistance for a two-point probe configuration can be estimated using the expression $R=F \rho / 2 a,{ }^{19}$ where $F$ is a thin film correction factor and $\rho / 2 a$ is the spreading resistance of a semi-infinite sample with $\rho$ the resistivity and $a$ the radius of the contact area. The thin film correction factor $F$ is given in Ref. 20 . Using $a \approx 50 \mathrm{~nm}$, the approximate size of the contact area, ${ }^{7}$ we estimated the correction factors: for $M 1, F \approx 5.61$ and for $M 2, F \approx 4.10$. Hence, the spreading resistance for our cases is found to be much smaller than $100 \Omega$. Thus, the contact resistance for the case of a gold electrode in contact with a gold film cannot be expected to contribute significantly to the measured two-point resistances. Furthermore, no dependence on contact pressure was observed.

We measured the two-point resistance of the Au pad using the two inner electrodes of a SiNW-4PP, N4 (m3). A mean value of $141.17 \pm 0.31 \Omega$ was obtained, which is around 15-30 $\Omega$ larger than similar measurements made with $\mu 4 \mathrm{PP}$. The discrepancy can be attributed to the fact that the SiNW electrodes give rise to an extra series resistance of roughly $15 \Omega$ for each of the two electrodes, as estimated from the approximate dimensions of the Au coating, $15 \times 0.3$ $\times 0.07 \mu \mathrm{m}$. In addition a slightly larger spreading resistance is expected due to a smaller contact area, but still the spreading resistance is expected to be small compared to the intrinsic probe series resistance.

On a $35 \mathrm{~nm}$ thick Au film (sample B) we performed four-point resistance measurements with three different SiNW probes as well as a $16 \mu \mathrm{m}$ spaced $\mu 4$ PP. In Table II we give both the measured resistance and the calculated resistivity using Eq. (1). Figure 3(a) shows the resistivity measured as a function of time, for the microprobe $M 1(m 6)$ as well as the three nanoprobes $N 1(\mathrm{~m} 7), N 2(\mathrm{~m} 8)$, and $N 3$ ( $m 9$ ). All measurements show a fluctuating signal around a rather constant average. We frequently observed the SiNW probes to snap in and out of contact, as shown for probe $N 3$ at $t=15 \mathrm{~s}$. The resistivity values found for the film were larger than the bulk resistivity of Au but did not show a clear dependence on the type of probe used. Comparing with the resistivity measured using $M 1$, there are about $10 \%-$ $30 \%$ variations in the measured resistivity for sample B using SiNW-4PPs.

Furthermore, we measured an Au film with a thickness of $7 \mathrm{~nm}$ (sample C). The results are given in Table II ( $m 10$ and $m 11$ ). The resistivity of the thin film, sample $\mathrm{C}$, is nearly a factor of 2 larger than the resistivity of sample B. The electrical resistivity of thin metallic films is known to increase with decreasing film thickness, due to increased interface scattering ${ }^{20,21}$ and grain-boundary scattering effects. ${ }^{22}$ In Fig. 3(b) we plotted the ratios of the measured resistivities for different samples to the bulk value of $\mathrm{Au}$ as a function of film thickness. Measurements on a $10.5 \mathrm{~nm}$ thick Au pad ( $\rho=5.13$ or $6.14 \mu \Omega \mathrm{cm}$ ) using the $\mu 4 \mathrm{PP}$ were added to the graph. No SiNW-4PP measurement was done on this sample.

Attekum and co-workers ${ }^{23}$ investigated the resistivity of thin $\mathrm{Au}$ films evaporated on $\mathrm{SiO}_{2}$ and found that for thin films in the $30 \mathrm{~nm}$ range, the average size of grains can be taken to be roughly equal to the film thickness, in accordance
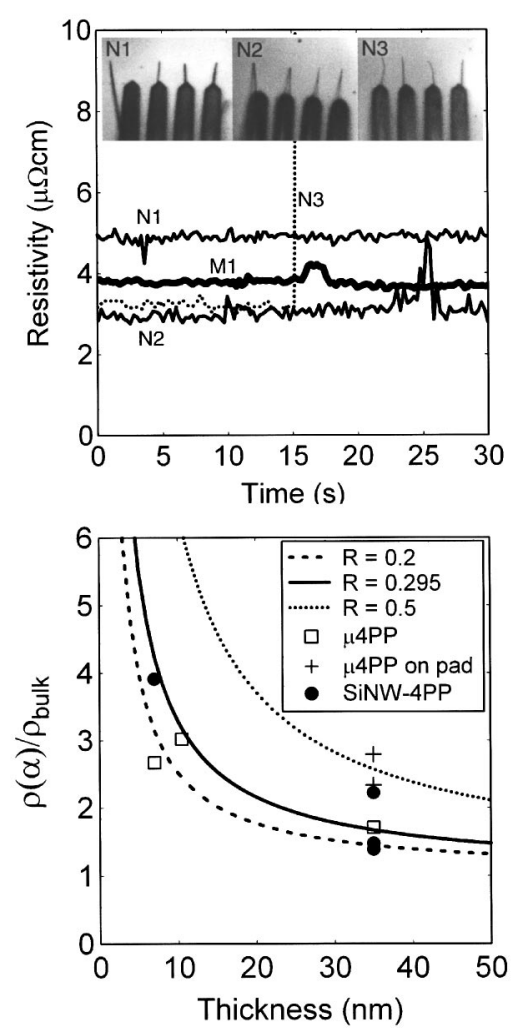

FIG. 3. (a) The inset shows optical images of the three SiNW probes, $N 1$, $N 2$, and $N 3$, used for measurement on sample B. The graph shows the resistivity vs time measured with the SiNW-4PP (thin lines) and the $\mu 4 \mathrm{PP}$ $M 1$ (thick lines) on $35 \mathrm{~nm}$ thick $\mathrm{Au}$ film. The shown measurements performed using probe $M 1, N 1, N 2$, and $N 3$ were numbered as $m 6, m 7, m 8$, and $m 9$, respectively, as listed in Table II. The measurement using $N 3$ (dotted line) was unstable and switched out of contact at $t=15 \mathrm{~s}$. With the SiNW-4PP, noise levels comparable to that of the $\mu 4 \mathrm{PP}$ measurement was achieved. (b) The ratios of the measured resistivity to the bulk value of Au are plotted for $7 \mathrm{~nm} \mathrm{Au}$ film and $35 \mathrm{~nm}$ Au film, with $\mu 4 \mathrm{PP}$ (open squares) and SiNW-4PP (filled circles). The crosses represent $\mu$ 4PP measurements on a $35 \mathrm{~nm} \mathrm{Au}$ pad. The solid line is a plot of the MS model with $R_{\mathrm{gr}}$ $=0.295 .{ }^{23}$ For comparison, curves calculated for $R_{\mathrm{gr}}=0.2$ (dashed line) and $R_{\mathrm{gr}}=0.5$ (dotted line) are plotted as well.

with Ref. 22. The increasing number of grain boundaries as the film thickness decreases, leads to an increase in the resistivity. In the MS theory, proposed by Mayadas and Shatzkes $(\mathrm{MS})^{22}$ the grain-boundary enhanced resistivity is given by

$$
\rho(\alpha) / \rho_{\text {bulk }}=\left[1-\frac{3}{2} \alpha+3 \alpha^{2}-3 \alpha^{3} \ln \left(1+\alpha^{-1}\right)\right]^{-1},
$$

with the bulk resistivity $\rho_{\text {bulk }}=2.2 \mu \Omega \mathrm{cm}$ and the parameter $\alpha=\left(\lambda_{\infty} / D_{\mathrm{gr}}\right)\left(R_{\mathrm{gr}} / 1-R_{\mathrm{gr}}\right)$. Here $\lambda_{\infty} \approx 44 \mathrm{~nm}$ is the electron mean free path in bulk gold, ${ }^{23} D_{\mathrm{gr}}$ is the mean grain diameter, and $R_{\mathrm{gr}}$ is the reflection coefficient for electrons striking the grain boundary. In Fig. 3(b) we plotted the calculated resistivity ratios vs film thickness based on the MS theory using $R_{\mathrm{gr}}=0.295$ (Ref. 23) as well as $R_{\mathrm{gr}}=0.2$ and 0.5. A reasonable agreement between the work done by Attekum and co-workers and our experimental results was obtained. The two measurements on Au pads are deviating considerably from the rest of the data; this could be due to added inaccuracies from the sample geometry factor. 


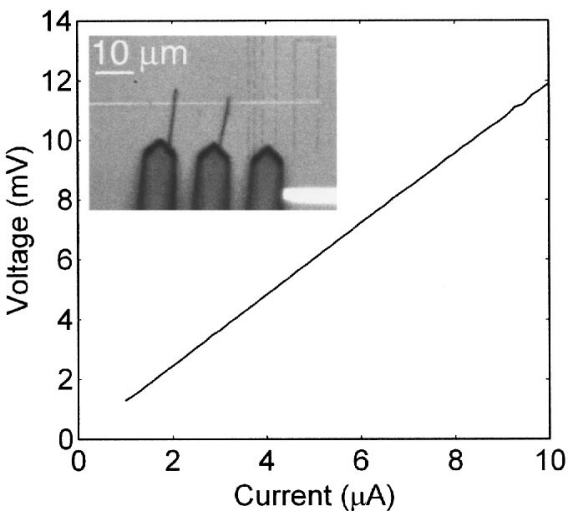

FIG. 4. $I-V$ characteristics of a $16 \mathrm{~nm}$ thick Au wire measured using a SiNW-based nano four-point probe in a two-point configuration. The inset is an optical image taken during the measurement (scale bar: $10 \mu \mathrm{m}$ ).

\section{MEASUREMENTS ON NANOWIRES}

The resistance of a $16 \mathrm{~nm}$ thick, $1 \mu \mathrm{m}$ wide Au nanowire (sample D) was measured using a SiNW-4PP in a two-point configuration, since the colinearity of the four electrode tips of the SiNW-4PPs was not sufficient to allow all four electrodes to be in contact with the nanowire at the same time.

All recorded $I / V$ curves show a linear behavior similar to the one displayed in Fig. 4. A mean resistance value of $1260 \pm 57 \Omega$ was obtained with a probe spacing of $16 \mu \mathrm{m}$, corresponding to a calculated resistivity of $121 \mu \Omega \mathrm{cm}$. If the measurement is corrected for a $140 \Omega$ intrinsic probe series resistance, the calculated resistivity becomes $108 \mu \Omega \mathrm{cm}$. This should be compared to the resistivity value of 94 $\mu \Omega \mathrm{cm}$ measured on the nanowire with an ordinary $\mu 4 \mathrm{PP}$ with $2 \mu \mathrm{m}$ electrode spacing used for a four-point resistance measurement. In the two-point resistance measurements with a SiNW-4PP spreading resistance effects are expected to contribute less than $10 \%$ to the measured resistance based on finite element modeling of the measurement. The measured resistivity on this film is consistently one order of magnitude higher than expected, the reason for this is unknown, but could be due to preparation details; island formation leading to a percolation like current flow pattern could be part of an explanation, which is supported by the fact that this film had a rough surface.

As mentioned in the Introduction, a micro four-point probe may damage fragile samples. After measurement, the contact points on the measured surface were inspected in a SEM. The SEM image in Fig. 5 shows the left SiNW electrode touching the surface of the Au nanowire. At a higher magnification, a mark of roughly $50 \mathrm{~nm}$ in size was found on the surface at the contact point, as indicated by the arrow in the left inset. Using the SiNW-4PP, the damage of the thin films was considerably reduced compared to the severe sample damage inflicted by the $\mu 4 \mathrm{PP}$ shown in the right inset of Fig. 5.

The contact force applied by a SiNW electrode can be estimated using the equation of the deflection force $F$ $=s 3 E I / l^{3}$, of a uniform cantilever of length $l$ with a force $F$ load at the free end. ${ }^{17}$ Here $E$ is the Young's modulus, $I$ the plane area moment of inertia, and $s$ the deflection of the

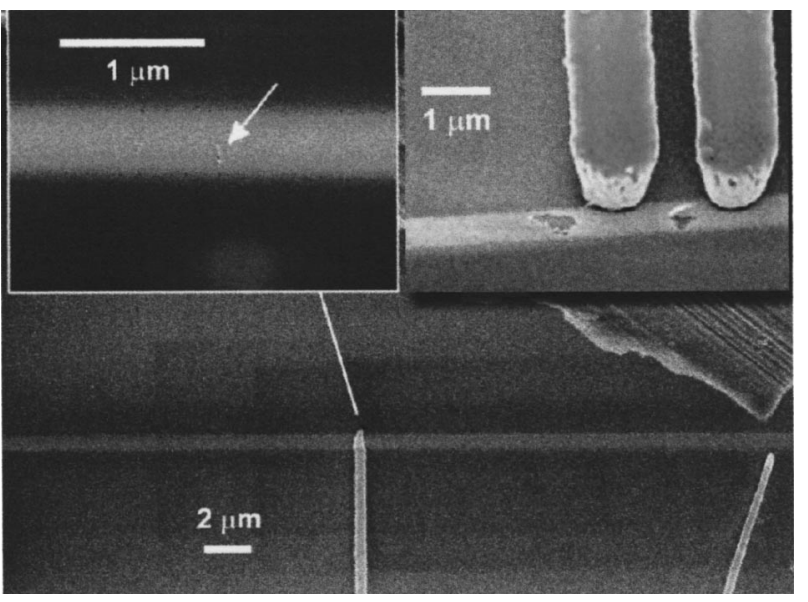

FIG. 5. SEM image showing one of the SiNW probes in contact with an Au nanowire (sample D). The SEM image in the left inset shows a scratch smaller than $50 \mathrm{~nm}$, caused by the mechanical contact between the SiNW electrode and the nanowire deposited on the surface. For comparison, the right inset shows a scratch made by a $\mu 4 \mathrm{PP}$ on a different but similar wire, with damaged areas in the $500 \mathrm{~nm}$ range.

cantilever. Because the nanowires were only partly covered with thin metal layers, it is difficult to accurately calculate the corresponding moment of inertia for the coated SiNW. To simplify the calculation, we could set the lower and upper limit for our case by calculating the contact force for an uncoated silicon wire as well as a silicon wire fully covered with two metal layers, $\mathrm{Ti}$ and $\mathrm{Au}$, assuming in both cases that the wire has a circular cross section. The contact force for a wire with two metallic layers is given by

$$
F=\frac{3\left(I_{\mathrm{SiNW}} E_{\mathrm{Si}}+I_{\mathrm{Au}} E_{\mathrm{Au}}+I_{\mathrm{Ti}} E_{\mathrm{Ti}}\right)}{l^{3}} s,
$$

with $\quad I_{\mathrm{SiNW}}=\pi d_{\mathrm{Si}}^{4} / 64, \quad I_{\mathrm{Ti}}=\pi\left(d_{\mathrm{Ti}}^{4}-d_{\mathrm{Si}}^{4}\right) / 64, \quad$ and $\quad I_{\mathrm{Au}}$ $=\pi\left(d_{\mathrm{Au}}^{4}-d_{\mathrm{Ti}}^{4}\right) / 64$, where $d_{\mathrm{si}}$ is the diameter of the SiNW, and $d_{\mathrm{Ti}}$ and $d_{\mathrm{Au}}$ are the outer diameter of the $\mathrm{Ti}$ and $\mathrm{Au}$ cylindrical layers, respectively. For a nanowire with a diameter of $200 \mathrm{~nm}$ and a length of $15 \mu \mathrm{m}$, a $500 \mathrm{~nm}$ deflection of the beam is sufficient to ensure a good electrical contact. This requires the force $F=4.5 \mathrm{nN}$ and $F=13 \mathrm{nN}$ for the pure and coated SiNW $\left(d_{\mathrm{Ti}}=210 \mathrm{~nm}, d_{\mathrm{Au}}=280 \mathrm{~nm}\right)$, respectively. In comparison, a standard $\mu 4 \mathrm{PP}$ will give a contact force in the order of $\mu \mathrm{N}$ for a deflection of a few hundred nanometers. Not only the contact area, but also the contact force can be dramatically reduced if the probe is reduced to nanoscale dimensions.

\section{DISCUSSION}

We have demonstrated that using silicon nanowires as templates for metal deposition, it is possible to fabricate narrow, soft multiprobes for electrical characterization of fragile thin films and nanostructures.

We compared measurement results obtained by microfabricated four-point probes to similar probes having such nanowire extensions. For thin Au film samples, the measured two-point resistance obtained with SiNW-4PP was slightly higher than for $\mu 4 \mathrm{PP}$, which could be roughly attributed to 
the resistance of the electrode gold layer on top of the SiNW electrodes. In four-point resistivity measurements, for all thin $\mathrm{Au}$ film samples studied here the SiNW probes showed variations of $10 \%-50 \%$ compared to the microprobes. The values measured for the resistivity could be explained using the MS model for grain-boundary scattering, and good agreement with experimental data reported by earlier studies ${ }^{23}$ was found.

On the $\mathrm{Au}$ nanowires, only two-point measurements were performed, due to the difficulty in obtaining sufficiently good alignment of the four electrode tips in the fabrication process, and thus achieving simultaneous electrical contact to the nanowires with all four SiNW electrodes. The high resistivity values found for the Au nanowire as compared to the bulk value cannot immediately be understood in terms of grain-boundary scattering. Durkan and Welland ${ }^{14}$ investigated the dependence of the resistivity on the width of $\mathrm{Au}$ nanowires with comparable thickness to ours, and found that the resistivity increases when the nanowire width becomes comparable to the grain size. Our Au nanowires have a significantly larger width $(1 \mu \mathrm{m})$ than thickness $(16 \mathrm{~nm})$, so we do not expect the width dependence to play a significant role for the surprisingly high measured resistivity. It is possible that the contact resistance is larger for the Au nanowires than for the thin Au film. To clarify this, four-point measurements on the Au nanowires must be performed with SiNW-4PP.

The fabrication method used has the advantage of allowing flexibility in the layout of the electrodes, since the length and spacing of the nanowire electrodes can be determined by individual manipulation. The method is for the same reason not suitable for volume production in its present form. Contact forces such as capillary and van der Waals forces aid in keeping the nanowire in stable positions on the microcantilevers, but also make submicron alignment of the electrode tips on the same axis difficult. The fine adjustment of the parts of the nanowires extending from the cantilevers is thus a main concern to be addressed in future experiments. A scheme for aligning of the four tips by pushing the four silicon nanowires against an edge of nonsticking material such as Teflon, prior to fixation by metallization, could be a solution to the problem. The assembly process could also be improved by modifying the geometrical structure of the cantilevers, such that the nanowires self-align, for instance, by incorporating a groove in the longitudinal direction of the cantilever, or by chemically modifying the surface of the microcantilever to self-center the nanowires. Alternatively, electron-beam lithography may be considered to fabricate nanoelectrode extensions of the $\mathrm{SiO}_{2}$ cantilevers.

Since the stiffness of electrodes decreases more rapidly with diameter than the contact area, surface adhesion forces, such as van der Waals and capillary forces, become important for such thin electrodes. Indeed, we observed frequently that the nanowire electrodes adhered to the surface when retracting. In some cases this even lead to the detachment of the nanowire electrode from the cantilever. Unless this problem is solved, we consider the SiNW-4PP unsuitable for scanning or mapping resistance values where repetitive measurements across a surface are required. ${ }^{8}$

\section{CONCLUSION}

In summary, we showed that the SiNW-4PP is useful for characterizing thin $\mathrm{Au}$ films and nanowires, and that the sample damage exerted on the Au nanowire sample could be considerably reduced using nanowire electrode extensions. One application for such probes, which has not yet been explored is local measurement of surface state conductivity of clean surfaces in ultrahigh vacuum, where minimal influence of the contacts on the surface properties is highly desired. $^{24}$

\section{ACKNOWLEDGMENTS}

We thank Erik Bakkers for providing silicon nanowires for the experiments and Dr. Martin Bammerlin and Dr. Reto R. Schlittler for providing nanostencils. The project was supported by the European Union in the fifth framework program project "ATOMS" (Grant No. IST-1999-14912).

${ }^{1}$ M. E. T. Molares, E. M. Hohberger, C. Schaeflein, R. H. Blick, R. Neumann, and C. Trautmann, Appl. Phys. Lett. 82, 2139 (2003).

${ }^{2}$ S. W. Chung, J. Y. Yu, and J. R. Heath, Appl. Phys. Lett. 76, 2068 (2000).

${ }^{3}$ J. F. Lin, J. P. Bird, L. Rotkina, and P. A. Bennett, Appl. Phys. Lett. 82, 802 (2003).

${ }^{4}$ A. Bietsch, M. A. Schneider, M. E. Welland, and B. Michel, J. Vac. Sci. Technol. B 18, 1160 (2000).

${ }^{5}$ A. Bietsch and B. Michel, Appl. Phys. Lett. 80, 3346 (2002).

${ }^{6}$ I. Shiraki, F. Tanabe, R. Hobara, T. Nagao, and S. Hasegawa, Surf. Sci. 493, 633 (2001).

${ }^{7}$ C. L. Petersen, T. M. Hansen, P. Boggild, A. Boisen, O. Hansen, T. Hassenkam, and F. Grey, Sens. Actuators, A 96, 53 (2002).

${ }^{8}$ P. Boggild, F. Grey, T. Hassenkam, D. R. Greve, and T. Bjornholm, Adv. Mater. Opt. Electron. 12, 947 (2000).

${ }^{9}$ I. Shiraki, T. Nagao, S. Hasegawa, C. L. Petersen, P. Boggild, T. H. Hansen, and F. Grey, Surf. Rev. Lett. 7, 533 (2000).

${ }^{10}$ S. Hasegawa, N. Sato, I. Shiraki, C. L. Petersen, P. Boggild, T. M. Hansen, T. Nagao, and F. Grey, Jpn. J. Appl. Phys., Part 1 39, 3815 (2000).

${ }^{11}$ P. Boggild, T. M. Hansen, O. Kuhn, F. Grey, T. Junno, and L. Montelius, Rev. Sci. Instrum. 71, 2781 (2000).

${ }^{12}$ J. Brugger, J. W. Berenschot, S. Kuiper, W. Nijdam, B. Otter, and M. Elwenspoek, Microelectron. Eng. 53, 403 (2000).

${ }^{13}$ R. Lin, M. Bammerlin, R. R. Schlittler, and P. Bøggild (unpublished).

${ }^{14}$ C. Durkan and M. E. Welland, Phys. Rev. B 61, 14215 (2000).

${ }^{15}$ J. E. A. M. van den Meerakker, R. J. G. Elfrink, F. Roozeboom, and J. F. C. M. Verhoeven, J. Electrochem. Soc. 147, 2757 (2000).

${ }^{16}$ M. Madou, Fundamentals of Microfabrication (CRC, Boca Raton, FL, 1997).

${ }^{17}$ Handbook of Physics, edited by W. Benenson, J. W. Harris, H. Stocker, and L. Holger (Springer, Berlin, 2000).

${ }^{18}$ C. L. Petersen, D. Worledge, and P. R. E. Petersen, in Spatially Resolved Characterization of Local Phenomena, Materials and Nanostructures, edited by D. A. Bonnell, J. Piqueras, A. P. Shreve, and F. Zypman, MRS Symposium Proceedings, No. 738 (Materials Research Society, Pittsburgh, 2003), p. 157.

${ }^{19}$ P. Blood and J. W. Orton, The Electrical Characterization of Semiconductors: Majority Carriers and Electron States (Academic, London, 1992).

${ }^{20}$ F. M. Smits, AT\&T Tech. J. 37, 711 (1958).

${ }^{21}$ E. H. Sondheimer, Adv. Phys. 1, 1 (1952).

${ }^{22}$ A. F. Mayadas, M. Shatzkes, and J. F. Janak, Appl. Phys. Lett. 14, 345 (1969).

${ }^{23}$ P. M. T. M. van Attekum, P. H. Woerlee, G. C. Verkade, and A. A. M. Hoeben, Phys. Rev. B 29, 645 (1984).

${ }^{24}$ C. L. Petersen, F. Grey, I. Shiraki, and S. Hasegawa, Appl. Phys. Lett. 77, 3782 (2000) 